INFECTION CONTROL AND HOSPITAL EPIDEMIOLOGY OCTOBER 2014, vol. 35 , NO. $\mathrm{S}_{3}$

\title{
Cost Savings of Universal Decolonization to Prevent Intensive Care Unit Infection: Implications of the REDUCE MRSA Trial
}

\author{
Susan S. Huang, MD, MPH;' Edward Septimus, MD; ${ }^{2}$ Taliser R. Avery, MPH; ${ }^{3}$ Grace M. Lee, MD, MPH; \\ Jason Hickok, MBA, RN; ${ }^{4}$ Robert A. Weinstein, MD $;{ }^{5}$ Julia Moody, MS; ${ }^{4}$ Mary K. Hayden, MD; ${ }^{6}$ \\ Jonathan B. Perlin, MD, PhD; ${ }^{4}$ Richard Platt, MD, MS; ${ }^{3}$ G. Thomas Ray, $\mathrm{MBA}^{7}$
}

\begin{abstract}
OBjEстіVE. To estimate and compare the impact on healthcare costs of 3 alternative strategies for reducing bloodstream infections in the intensive care unit (ICU): methicillin-resistant Staphylococcus aureus (MRSA) nares screening and isolation, targeted decolonization (ie, screening, isolation, and decolonization of MRSA carriers or infections), and universal decolonization (ie, no screening and decolonization of all ICU patients).
\end{abstract}

DESIGN. Cost analysis using decision modeling.

METHODS. We developed a decision-analysis model to estimate the health care costs of targeted decolonization and universal decolonization strategies compared with a strategy of MRSA nares screening and isolation. Effectiveness estimates were derived from a recent randomized trial of the 3 strategies, and cost estimates were derived from the literature.

RESULTS. In the base case, universal decolonization was the dominant strategy and was estimated to have both lower intervention costs and lower total ICU costs than either screening and isolation or targeted decolonization. Compared with screening and isolation, universal decolonization was estimated to save $\$ 171,000$ and prevent 9 additional bloodstream infections for every 1,000 ICU admissions. The dominance of universal decolonization persisted under a wide range of cost and effectiveness assumptions.

CONCLUSIONs. A strategy of universal decolonization for patients admitted to the ICU would both reduce bloodstream infections and likely reduce healthcare costs compared with strategies of MRSA nares screening and isolation or screening and isolation coupled with targeted decolonization.

Infect Control Hosp Epidemiol 2014;35(S3):S23-S31

The intensive care unit (ICU) has been a long-standing focus of attention for reducing largely preventable healthcareassociated infections. ${ }^{1-3}$ Many prevention strategies have emerged to reduce the prevalence or transmission of antibiotic-resistant pathogens, such as methicillin-resistant Staphylococcus aureus (MRSA), as well as to reduce bloodstream infections (BSIs). ${ }^{4-7}$ Several of these strategies have evidence of benefit in observational studies, but comparative effectiveness studies have only recently been published, and comparative cost analyses are lacking. ${ }^{8-11}$

Recently, 3 promising strategies were compared in a large, cluster-randomized trial (the Randomized Evaluation of Decolonization versus Universal Clearance to Eliminate [REDUCE] MRSA trial). ${ }^{9}$ This 43-hospital, 74-ICU trial compared the effectiveness of screening and isolation, targeted decolonization, and universal decolonization with respect to their ability to reduce the rate of MRSA-positive clinical cultures and all-pathogen BSIs in adults. "Screening and isolation" was the routine and long-standing strategy in these hospitals and consisted of screening all patients for MRSA nasal colonization at admission to the ICU and using contact precautions for patients with current or previous MRSA colonization or infection. ${ }^{12}$ One-third of hospitals were randomized to initiate "targeted decolonization," which involved screening and isolation but added a 5-day decolonization regimen for all known MRSA carriers or patients with MRSA infection that consisted of twice-daily intranasal mupirocin and daily bathing with chlorhexidine-impregnated cloths. Another third of hospitals were randomized to initiate "universal decolonization," in which ICU admission screening for MRSA

Affiliations: 1. Division of Infectious Diseases and Health Policy Research Institute, University of California Irvine School of Medicine, Orange, California; 2. Hospital Corporation of America and Texas A\&M Health Science Center College of Medicine, Houston, Texas; 3. Department of Population Medicine, Harvard Pilgrim Health Care Institute and Harvard Medical School, Boston, Massachusetts; 4. Hospital Corporation of America, Nashville, Tennessee; 5. Department of Medicine, Cook County Health and Hospitals System, Chicago, Illinois; 6. Department of Pathology and Laboratory Medicine, Rush University Medical Center, Chicago, Illinois; 7. Division of Research, Kaiser Permanente Medical Care Program, Northern California Region, Oakland, California.

Received March 16, 2014; accepted April 11, 2014; electronically published September 15, 2014.

(C) 2014 by The Society for Healthcare Epidemiology of America. All rights reserved. 0899-823X/2014/35S3-0005\$15.00. DOI: 10.1086/677819 
was stopped, contact precautions were unchanged from the other strategies, and all patients received twice-daily intranasal mupirocin for 5 days plus daily bathing with chlorhexidine-impregnated cloths for the entire ICU stay.

The results of the REDUCE MRSA trial indicated that universal decolonization was more effective than targeted decolonization or screening and isolation in reducing BSIs from any pathogen. However, the cost implications of these strategies have not been addressed in light of these results. Our objective was to estimate the incremental effect on healthcare costs associated with targeted decolonization and universal decolonization compared with screening and isolation, which we considered to be the current standard of care. If either of these 2 strategies were found to be less costly than screening and isolation, the benefits of implementing one of these strategies would be to not only improve health but also decrease use of healthcare resources.

\section{METHODS}

\section{Decision Analysis Model}

We developed a static decision analysis model, programmed in Microsoft Excel, that compared the effects of the above 3 strategies for preventing infections in adult ICUs: (1) screening and isolation, (2) targeted decolonization, and (3) universal decolonization (Table 1). ${ }^{9}$

Using data from the REDUCE MRSA trial and cost and utilization estimates from published sources, we simulated the effects of each of the 3 strategies on healthcare costs, ICU days, and BSIs for 1 year, assuming a hospital with 1,000 annual ICU admissions. Because the trial did not measure effects outside of the ICU, we only modeled ICU impact. The primary perspective was that of the healthcare system. Because this was strictly a cost analysis, no quality-of-life measures were incorporated, nor were health states assigned dollar values.

\section{Model Inputs: Percentage of Patients Placed on Contact Precautions}

We assumed that the background percentage of patients who arrive at the ICU and have test results positive for MRSA or have a known recent history of MRSA infection or coloni- zation was $11 \%$ (Table 2). This estimate was based on the ICU MRSA importation rate reported from the baseline period in all arms of the REDUCE MRSA trial, ${ }^{9}$ and it remained unchanged during the implementation phase for the arms that continued screening. For the universal decolonization arm, knowledge of MRSA importation decreased in the implementation phase to $4 \%$, likely because of cessation of screening and possibly because of effects of decolonization for patients readmitted to the ICU. Therefore, under the universal decolonization strategy, fewer persons were modeled as being placed on contact precautions. For this cost analysis, it was not necessary to model the "true" percentage of patients colonized with MRSA or the relationship between MRSA colonization and the rate of BSI. The percentage of patients with test results positive for MRSA is primarily important in the model because it affects the number of persons who are placed on contact precautions and therefore affects costs. For the universal colonization strategy, $4 \%$ of patients were placed on contact precautions (and therefore incurred the associated costs) because they had some known history of MRSA colonization or infection, but this percentage should not be interpreted as directly relating to any "true" colonization rate.

\section{Model Inputs: Risk of BSI}

Using data from the REDUCE MRSA trial, the baseline incidence of BSI was assumed to be 19.63 cases per $1,000 \mathrm{ICU}$ admissions, equivalent to approximately 5 infections per 1,000 ICU-days (Table 2). The risk of BSI under the 3 different strategies was also derived from the REDUCE MRSA trial, which reported as-assigned adjusted hazard ratios of 0.98 , 0.77 , and 0.55 for the screening and isolation, targeted decolonization, and universal decolonization strategies, respectively. ${ }^{9}$ These results were relative to the pre-trial time period, in which all arms were performing screening and isolation. To compare the targeted decolonization and universal decolonization strategies to screening and isolation, we adjusted the results of those strategies so as to be relative to the longstanding gold standard of screening and isolation. ${ }^{12}$ This resulted in the relative risk of BSI being 1.00, 0.7857, and 0.5612 for the screening and isolation, targeted decolonization, and universal decolonization strategies, respectively.

TA BLE 1. Description of the Strategies to Prevent Intensive Care Unit (ICU) Bloodstream Infection Considered in the Cost Analysis Strategy Description

Screening and isolation baseline strategy

Universal nares screening for MRSA carriage at ICU admission; use of contact precautions for MRSA carriers (by history or positive screening test result/clinical culture)

Targeted decolonization

Universal nares screening for MRSA carriage at ICU admission; use of contact precautions for MRSA carriers (by history or positive screening test result/clinical culture); decolonize MRSA carriers with daily chlorhexidine baths and mupirocin treatment

Universal decolonization

No screening for MRSA at admission to the ICU; use of contact precautions for MRSA carriers (by history or positive clinical culture); give daily chlorhexidine baths and mupirocin to all patients in ICU

NOTE. MRSA, methicillin-resistant Staphylococcus aureus. 
TA BLE 2. Decision-Analysis Model Inputs Derived from the REDUCE MRSA Trial: MRSA Colonization and Risk of Bloodstream Infection by Intensive Care Unit (ICU) Strategy

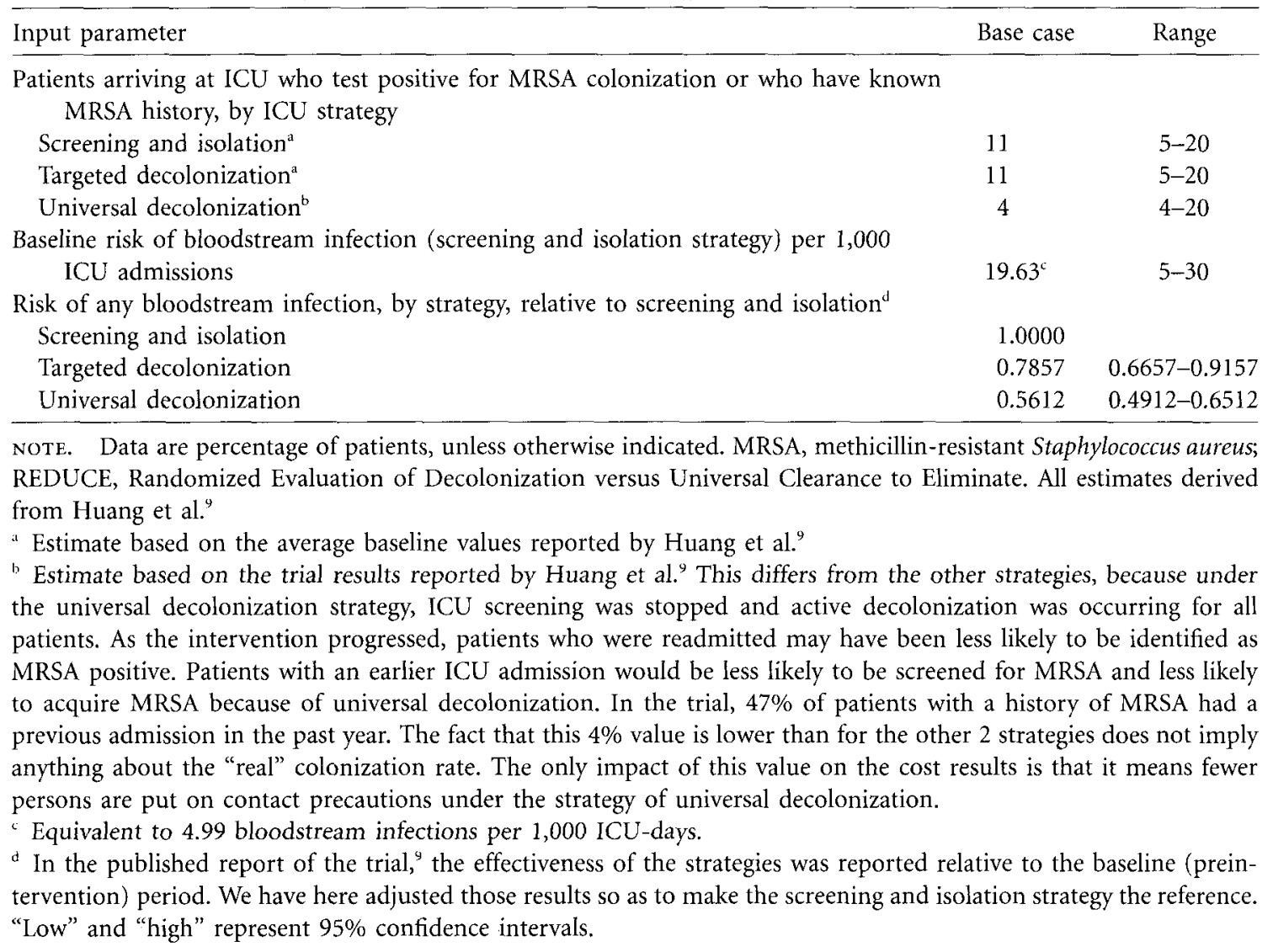

\section{Model Inputs: Costs and ICU-Days}

Parameter estimates related to the base case are shown in Table 3. Intervention costs were derived from the literature. Intervention-related costs are the costs of screening, contact precautions, and decolonization. Where possible, preference was given to estimates that were more recent and were from the United States. The estimates used in most of the recent literature did not vary substantially, with the exception of the cost of an MRSA screening test and the cost of mupirocin. Variation in screening costs were primarily related to whether the screening was assumed to be performed using the more costly polymerase chain reaction (PCR) test or the less costly chromogenic agar culture. Variation in literature estimates of the cost of mupirocin primarily related to whether generic or trade mupirocin was assumed to be used. In our base case, we assumed the chromogenic agar culture was used for screening (as it was in nearly all hospitals of the REDUCE MRSA trial) and that generic mupirocin was used for decolonization. We also assumed that positive chromogenic agar cultures would not require confirmatory tests, and therefore we assumed no difference in screening costs between positive and negative screens. Any additional labor costs involved in applying mupirocin were assumed to be negligible, and because patients are typically bathed at least once a day as part of usual care, we assumed that the cost of bathing with chlorhexidine cloths does not require any additional labor. Cost estimates reflect the "steady state;" we did not attempt to estimate start-up costs, such as those that might be associated with training staff. All cost estimates are in \$US, adjusted to year 2012 using the consumer price index.

We used estimates from the literature to estimate the incremental hospital days and cost of a BSI (and therefore the incremental savings associated with preventing an infection). Hassan et $\mathrm{al}^{13}$ estimated that the incremental hospital-days associated with a hospital-acquired infection (not specifically BSI) was 3.296. In addition, they estimated that the cost of an additional hospital-day once an infection was acquired was $24 \%$ higher than the cost of an uninfected day. Dasta et $a^{16}$ estimated that the mean cost of an ICU-day (after day 3) was $\$ 4,385$ (after adjusting to 2012 dollars). Applying the above estimates, we estimated the incremental cost of a BSI to be $\$ 17,920(3.296 \times[4,385+(0.24 \times 4,385)])$. This estimate is consistent with the estimated incremental cost of BSIs reported by Kilgore et $\mathrm{al}^{25}$ ( $\$ 22,371$ in year 2012 dollars), who, like Hassan et $\mathrm{al}^{13}$, used a large US database and implemented methods to control for heterogeneity and endogeneity biases. All differences among the 3 strategies in hospital days and general ICU costs (not including intervention 
Table 3. Decision-Analysis Model Inputs and Literature Sources, Base Case Scenario

\begin{tabular}{|c|c|c|c|}
\hline Input parameter & Base case & $\begin{array}{l}\text { Range for } \\
\text { sensitivity analyses }\end{array}$ & $\begin{array}{l}\text { Sources for } \\
\text { base case, range }\end{array}$ \\
\hline Cost of screening test $(\$)$ & 9.12 & $9.12-48.24$ & Fee schedule, fee schedule ${ }^{3}$ \\
\hline Cost per gown $(\$)$ & 0.96 & & Kang et $\mathrm{al}^{21}$ \\
\hline Cost per pair of gloves $(\$)$ & 0.09 & & Kang et $\mathrm{al}^{21}$ \\
\hline Time to don and doff gown and gloves (minutes) & 1.00 & $1.00-2.00$ & Kang et $\mathrm{a}^{21}$; Puzniak et $\mathrm{a}^{2^{22}}$ \\
\hline Hourly wage, nurses & 34.24 & & Kang et $\mathrm{al}^{21}$ \\
\hline Hourly wage, physicians & 89.46 & & Kang et $\mathrm{al}^{21}$ \\
\hline Total cost per day of contact precautions & 118.84 & & Calculated \\
\hline \multicolumn{4}{|l|}{ Decolonization } \\
\hline Cost of chlorhexidine impregnated cloths, 1 body wash (\$) & 5.35 & & Nelson et $\mathrm{al}^{15}$ \\
\hline Mean ICU-days for patients with negative MRSA screen without bloodstream infection ${ }^{f}$ & 4.15 & & Trial data \\
\hline Mean ICU-days for patients with positive MRSA screen without bloodstream infection ${ }^{f}$ & 5.15 & & Trial data \\
\hline $\begin{array}{l}\text { Incremental ICU-days for persons with bloodstream infection, but due to additional } \\
\text { comorbidities rather than infection per } \mathrm{se}^{\mathrm{B}}\end{array}$ & 3.46 & & Hassan et $\mathbf{a}^{13}$ \\
\hline Mean incremental ICU-days due to bloodstream infection ${ }^{B}$ & 3.30 & & Hassan et $\mathrm{al}^{13}$ \\
\hline Cost per ICU-day, days without bloodstream infection $(\$)^{\mathrm{h}}$ & 4,385 & & Dasta et $\mathrm{al}^{16}$ \\
\hline Incremental cost per ICU-day for patients with bloodstream infection $(\$)^{\mathrm{i}}$ & 1,052 & & Hassan et $\mathrm{al}^{13}$; Dasta et $\mathrm{al}^{16}$ \\
\hline Total incremental cost of bloodstream infection $(\$)^{i}$ & 17,920 & $2,880-35,000$ & $\begin{array}{l}\text { Calculated; Roberts et al }{ }^{14} \\
\text { Kilgore and Brossette }\end{array}$ \\
\hline
\end{tabular}

Note. All costs are in \$US and have been adjusted to the 2012 price level using the consumer price index. ICU, intensive care unit; PCR, polymerase chain reaction.

"Base case assumes screening culture using chromogenic agar, assuming no extra cost for a positive result. High-range cost assumes use of PCR screening test. Cost estimates are national limit reimbursement from 2013 US clinical diagnostic laboratory fee schedule, available at http://www.cms.gov/Medicare/ Medicare-Fee-for-Service-Payment/ClinicalLabFeeSched/clinlab.html, HCPCS 87081, "Culture screen only," and HCPCS 87641 "Mr-staph dna amp probe." ' Base case visit counts were based on direct observation of staff at 3 Department of Veterans Affairs hospitals and 1 university teach hospital. ${ }^{23}$ The actual observed mean physician visits per ICU-day was 17 , but it was estimated that approximately $25 \%$ of these visits were associated with the teaching function of the hospitals (Daniel Morgan, personal communication). We therefore assumed 13 mean physician visits per day.

"Assuming 1 bath per day as routine, plus 0.25 baths per day to account for large patients, incontinence, and wastage.

'Base case assumes use of generic mupirocin. High value is based on authors' analysis of average wholesale price for trade mupirocin.

"Assume negligible additional labor time for applying mupirocin and that use of chlorhexidine cloths requires no additional time relative to normal bath.

Estimates are from unpublished data from the Randomized Evaluation of Decolonization versus Universal Clearance to Eliminate [REDUCE] MRSA trial. ${ }^{9}$ These lengths of stay were applied to the screening and isolation and targeted decolonization strategies. For universal decolonization, in which patients are not screened for MRSA colonization, the length of stays were calibrated so as to be the same as stays in the other 2 strategies, except for any reductions caused by prevention of bloodstream infections.

${ }^{2}$ Estimates reported in Hassan et al ${ }^{13}$ were for all hospital-acquired infections and all units. We assumed these would apply to bloodstream infections that occurred in the ICU and that the additional hospital-days would be in the ICU.

"Because we use this estimate for the incremental costs of additional days within a stay, we used the mean cost as reported by Dasta et al ${ }^{16}$ for days 3 and later. The mean cost per ICU-day reported by Dasta et al ${ }^{16}$ was not specifically for uninfected days. However, given the relatively low incidence of hospitalacquired infections (about $6 \%$ as reported in Hassan et $\mathrm{al}^{13}$ ), we treated this as a reasonable estimate for the cost of an uninfected ICU-day.

Percentage increase $(24 \%)$ in uninfected cost per day as estimated by Hassan et $\mathbf{a}^{13}$ applied to estimated cost per ICU-day of Dasta et al. ${ }^{16}$

The low value of $\$ 2,880$ is $16 \%$ of the base case estimate and is an estimate of short-run marginal costs that includes only healthcare worker supplies.

costs) were assumed to be due entirely to differences in the incidence of BSIs and their effects on length of stay and cost per day.

To test the sensitivity of the model results, we varied several important parameters (baseline incidence of BSI, reduction in BSI associated with universal decolonization, and incre- mental ICU cost of BSI) over a wide range. In addition, we estimated the differences between the 3 screening strategies under a number of alternative scenarios to the base case. For example, we included a scenario in which the PCR test, rather than chromogenic culture, is used to test for MRSA, and a scenario in which trade, rather than generic, mupirocin is 
TABLE 4. Costs, Length of Stay, and Cost Savings Associated with 3 Intensive Care Unit (ICU) Strategies to Reduce ICU Bloodstream Infection per 1,000 ICU Admissions, Base Scenario

\begin{tabular}{|c|c|c|c|}
\hline \multirow[b]{2}{*}{ Variable } & \multicolumn{3}{|c|}{ Strategy } \\
\hline & $\begin{array}{c}\text { Baseline } \\
\text { screening } \\
\text { and isolation }\end{array}$ & $\begin{array}{c}\text { Targeted } \\
\text { decolonization }\end{array}$ & $\begin{array}{c}\text { Universal } \\
\text { decolonization }\end{array}$ \\
\hline \multicolumn{4}{|l|}{ ICU admissions } \\
\hline Total admissions to ICU & 1,000 & 1,000 & 1,000 \\
\hline No. of patients screened for MRSA at admission & 1,000 & 1,000 & 0 \\
\hline $\begin{array}{l}\text { No. of patients screening positive for MRSA (or } \\
\text { status is otherwise known at admission) }\end{array}$ & 110 & 110 & 40 \\
\hline No. of patients on contact precautions & 110 & 110 & 40 \\
\hline No. of patients decolonized & 0 & 110 & 1,000 \\
\hline No. of patients with any bloodstream infection & 20 & 15 & 11 \\
\hline \multicolumn{4}{|l|}{ ICU-days } \\
\hline Total no. of ICU-days & 4,391 & 4,377 & 4,362 \\
\hline No. of patient-days with contact precautions & 581 & 579 & 209 \\
\hline \multicolumn{4}{|l|}{ Costs $(\$)$} \\
\hline Cost of MRSA screening tests & 9,120 & 9,120 & 0 \\
\hline Cost of contact precautions & 69,010 & 68,830 & 24,890 \\
\hline Cost of decolonization & 0 & 4,679 & 36,500 \\
\hline $\begin{array}{l}\text { ICU costs, not including screening, contact pre- } \\
\text { cautions and decolonization }\end{array}$ & $19,320,000$ & $19,250,000$ & $19,170,000$ \\
\hline Total cost & $19,400,000$ & $19,330,000$ & $19,230,000$ \\
\hline Total cost per admission & 19,400 & 19,330 & 19,230 \\
\hline \multicolumn{4}{|l|}{$\begin{array}{l}\text { Incremental costs (savings) per admission com- } \\
\text { pared with screening and isolation (\$) }\end{array}$} \\
\hline $\begin{array}{l}\text { Intervention costs only (screening, contact pre- } \\
\text { cautions, decolonization) }\end{array}$ & $\cdots$ & 4 & (17) \\
\hline Bloodstream infection-related ICU costs & $\ldots$ & $(75)$ & $(155)$ \\
\hline All costs & $\ldots$ & $(71)$ & $(171)$ \\
\hline \multicolumn{4}{|l|}{$\begin{array}{l}\text { Incremental costs (savings) per admission com- } \\
\text { pared with screening and isolation (\$) }\end{array}$} \\
\hline $\begin{array}{l}\text { Intervention costs only (screening, contact pre- } \\
\text { cautions, decolonization) }\end{array}$ & $\cdots$ & $\cdots$ & (21) \\
\hline Bloodstream infection-related ICU costs & $\ldots$ & $\ldots$ & $(79)$ \\
\hline All costs & ... & $\ldots$ & $(100)$ \\
\hline
\end{tabular}

NOTE. MRSA, methicillin-resistant Staphylococcus aureus.

used for decolonization. Another scenario included only those costs estimated to be variable in the short run. In their study of variable versus fixed costs of hospital care, Roberts et al ${ }^{14}$ treated labor and overhead as fixed in the short run and estimated that only $16 \%$ of all hospital costs (corresponding to healthcare worker supplies) were avoidable in the short run. Therefore, in this scenario, we assumed that the incremental cost of a BSI was $16 \%$ of our base case estimate, and we assumed no incremental cost for labor to don and doff gowns and gloves.

\section{RESULTS}

Over the course of 1 year at a hospital with 1,000 annual adult ICU admissions, we estimated that a strategy of universal decolonization would prevent 9 BSIs compared with a strategy of screening and isolation (11 vs 20) and 4 BSIs compared with a strategy of targeted decolonization (11 vs
15; Table 4). The universal decolonization strategy was estimated to have the lowest mean intervention costs, saving $\$ 17$ per admission compared with screening and isolation and saving \$21 per admission compared with targeted decolonization. By preventing BSIs, the universal decolonization strategy was estimated to save $\$ 155$ per admission in nonintervention, ICU-related costs compared with targeted decolonization. In total, for every ICU admission, universal decolonization was estimated to save $\$ 171$ compared with screening and isolation and $\$ 100$ compared with targeted decolonization.

These results were robust to changes in the parameter estimates that were derived from the REDUCE MRSA trial. ${ }^{9}$ Figure $1 A$ shows the estimated savings per admission (compared with screening and isolation) of universal decolonization, assuming it was less effective at preventing BSIs than was reported in the REDUCE MRSA trial. Because the strategy 
A

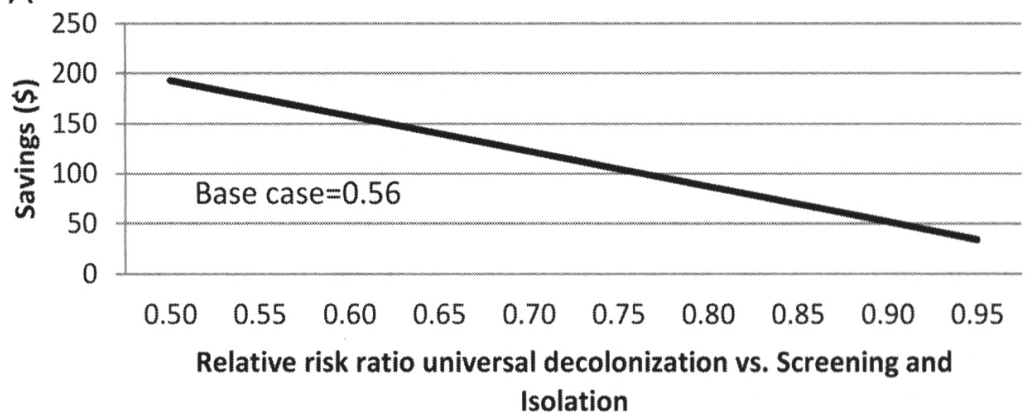

B

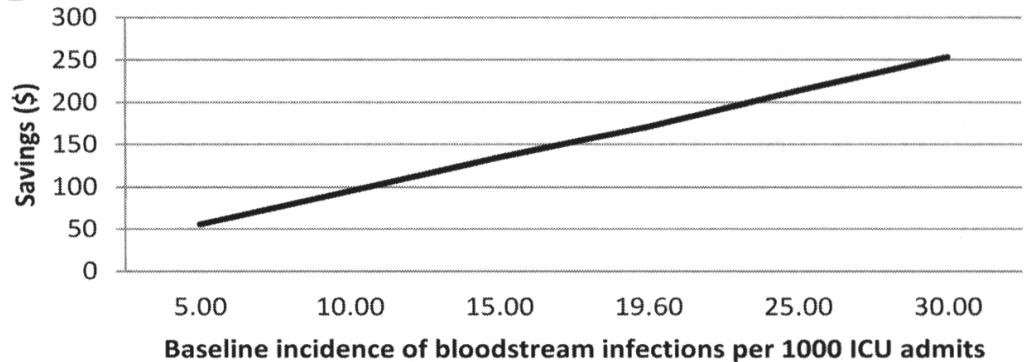

C

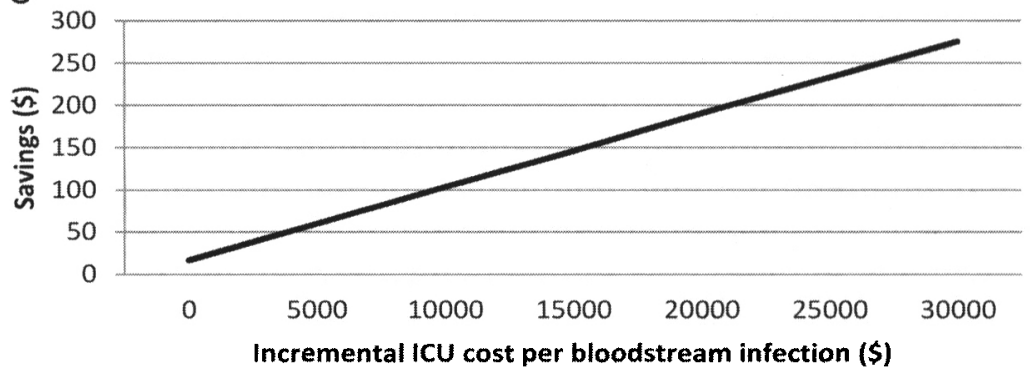

FIGURE 1. One-way sensitivity analyses of the estimated cost savings of universal decolonization compared with screening and isolation per intensive care unit (ICU) admission when evaluating $(A)$ the relative reduction (risk ratio) in bloodstream infections, $(B)$ the baseline incidence of ICU bloodstream infections, and $(C)$ the incremental cost of an ICU bloodstream infection.

of universal decolonization was estimated to have lower intervention costs than strategies involving screening, we estimated there would be modest cost savings even if it were not more effective in preventing BSIs than screening and isolation alone. Results were also robust to varying assumptions regarding the baseline incidence of ICU BSIs (Figure $1 B$ ) and to the incremental cost of a BSI (Figure $1 C$ ).

We estimated the differences between the 3 screening strategies under a number of alternative scenarios to the base case (Table 5). Results were robust to a large number of plausible alterations in the assumptions. For example, if the more costly PCR test is used for MRSA screening, the universal decolonization strategy saves $\$ 210$ per ICU admission versus $\$ 171$ if chromogenic agar culture is used (scenario 1). If the more costly trade mupirocin is used for decolonization, the universal decolonization strategy saves $\$ 85$ per ICU admission versus $\$ 171$ if generic mupirocin is used (scenario 2). When including only costs estimated to be avoidable in the short- term (ie, when all labor is considered to be a "fixed" cost in the time period under consideration), universal decolonization saves $\$ 23$ per ICU admission (scenario M1) compared with screening and isolation. Only under the most pessimistic combination of assumptions did the universal colonization strategy cost more than the other two strategies.

\section{DISCUSSION}

Universal decolonization has been shown to be a more effective strategy for reducing BSIs due to all pathogens than either MRSA screening and isolation or MRSA screening plus targeted decolonization. ${ }^{9}$ We now show that universal decolonization is also likely to reduce costs. Because savings come both from reductions in BSIs (and associated ICU savings) and from lower intervention costs, the benefits of universal decolonization are robust across a wide range of plausible cost and effectiveness assumptions. Consistent with 
TABLE 5. Sensitivity Analyses of Cost Savings of 3 Intensive Care Unit (ICU) Strategies to Reduce ICU Bloodstream Infection per 1,000 ICU Admissions

\begin{tabular}{|c|c|c|}
\hline \multirow[b]{2}{*}{ Scenario number and description } & \multicolumn{2}{|c|}{$\begin{array}{l}\text { Cost (saving) } \\
\text { per admission of } \\
\text { universal decolonization, \$US }\end{array}$} \\
\hline & $\begin{array}{l}\text { Compared with } \\
\text { screening and isolation }\end{array}$ & $\begin{array}{l}\text { Compared with } \\
\text { targeted decolonization }\end{array}$ \\
\hline Base case & $(171)$ & $(100)$ \\
\hline \multicolumn{3}{|l|}{ One-way sensitivity } \\
\hline 1. Assume MRSA screening is done using polymerase chain reaction test ( $\$ 48)$ & $(210)$ & $(140)$ \\
\hline 2. Assume use of trade mupirocin $(\$ 94)$ & $(85)$ & $(23)$ \\
\hline 3. Assume time to don and doff gloves and gown is 2 minutes & $(190)$ & (119) \\
\hline 4. Assume 30 nurse and 6 physician visits per ICU-day & $(151)$ & $(80)$ \\
\hline 5. Assume no incremental labor costs associated with contact precautions & $(153)$ & $(82)$ \\
\hline $\begin{array}{l}\text { 6. Assume } 20 \% \text { of patients arriving at ICU under screening and isolation and } \\
\text { targeted decolonization strategies have history of MRSA and are placed on } \\
\text { contact precautions }\end{array}$ & (228) & $(160)$ \\
\hline $\begin{array}{l}\text { 7. Assume } 5 \% \text { of patients arriving at ICU under screening and isolation and } \\
\text { targeted decolonization strategies have history of MRSA and are placed on } \\
\text { contact precautions }\end{array}$ & (134) & $(60)$ \\
\hline $\begin{array}{l}\text { 8. Assume } 10.8 \% \text { of patients arriving at ICU under universal decolonization } \\
\text { have history of MRSA and are placed on contact precautions (ie, the same } \\
\text { percent as for the other strategies) }\end{array}$ & $(128)$ & $(57)$ \\
\hline $\begin{array}{l}\text { 9. Assume incremental cost of bloodstream infection is } \$ 2,880 \text { (estimate of } \\
\text { short-run variable costs) }\end{array}$ & $(41)$ & $(34)$ \\
\hline 10. Assume incremental cost of bloodstream infection is $\$ 35,000$ & $(299)$ & $(166)$ \\
\hline $\begin{array}{l}\text { 11. Assume relative risk of bloodstream infection in universal decolonization } \\
\text { strategy compared with screening and isolation strategy is } 0.4912 \text { (rather } \\
\text { than } 0.5612 \text { ) }\end{array}$ & $(196)$ & $(125)$ \\
\hline $\begin{array}{l}\text { 12. Assume relative risk of bloodstream infection in universal decolonization } \\
\text { strategy compared with screening and isolation strategy is } 0.6512 \text { (rather } \\
\text { than } 0.5612 \text { ) }\end{array}$ & $(140)$ & $(69)$ \\
\hline \multicolumn{3}{|l|}{ Multiway sensitivity } \\
\hline $\begin{array}{l}\text { M1. Include only those costs estimated to be short-run variable costs (scenar- } \\
\text { ios } 5 \text { and 9) }\end{array}$ & $(23)$ & $(16)$ \\
\hline $\begin{array}{l}\text { M2. Combine assumptions least favorable to universal decolonization strategy } \\
(\text { scenarios } 2,5,8,9 \text {, and } 12)^{a}\end{array}$ & 94 & 92 \\
\hline $\begin{array}{l}\text { M3. Combine assumptions most favorable to universal decolonization strategy } \\
\text { (scenarios } 1,4,6,10 \text {, and } 11)\end{array}$ & $(507)$ & $(367)$ \\
\hline
\end{tabular}

Nоте. Data differ from those given in the text due to rounding; data in parentheses are savings. MRSA, methicillin-resistant Staphylococcus aureus.

a Scenario 7 was excluded because it was considered implausible for the universal decolonization strategy to have a higher percentage of patients on contact precautions given that no MRSA testing is performed in this strategy.

other studies, ${ }^{15}$ we also found that a strategy of screening and targeted decolonization, although not as cost-beneficial as universal decolonization, also resulted in lower costs and fewer BSIs than screening and isolation alone.

For a hospital with 1,000 ICU admissions per year, we estimate that universal decolonization would prevent 9 BSIs and potentially save approximately $\$ 171,000$ annually compared with screening and isolation. The majority of the estimated savings $(\$ 155,000)$ are associated with expected reductions in BSIs. Because the combined cost of chlorhexidine-impregnated cloths and generic mupirocin is similar to the cost of screening cultures, the balance of the savings
$(\$ 17,000)$ is primarily related to savings from reducing the number of patients placed on contact precautions. Using our base case cost assumptions, intervention costs will be similar under both strategies only if the percentage of patients placed on contact precautions is similar, which is highly unlikely given the lack of screening testing under universal decolonization, or if we assume no incremental labor costs for donning gloves and gowns.

A strategy of universal decolonization has been shown to reduce BSIs by over $40 \%,{ }^{9}$ and most of the savings from implementing that strategy would come from reducing BSIs and the associated ICU-days. The magnitude of the actual 
savings at any given institution will largely depend on the ability to realize cost reductions from fewer infections. Using estimates from the literature, we assumed that each infection prevented would avert 3.3 ICU-days and potentially save $\$ 18,000$. The estimated cost per ICU-day that we used was based on hospital charges that were then adjusted downward by cost-to-charge ratios. ${ }^{16}$ As such, it may be thought of as an estimate of long-run average cost. For any given hospital, the savings from averting infections is likely to be substantially less and will depend, for example, on staffing flexibility and the value of alternative uses for the freed-up bed-days. ${ }^{17,18}$

Nevertheless, the cost-savings of the universal decolonization strategy should be realized even by those institutions with excess capacity or staffing that is hard to reduce in the short term. Even when using a very conservative estimate of variable costs (one not including any labor costs), universal decolonization still dominates the other 2 strategies. We note also that our analysis is otherwise conservative in that we have not included any productivity or time costs associated with reduced morbidity.

In addition, because overall intervention costs were similar or lower in our base case estimates, the universal decolonization strategy was the most cost-saving strategy, even under assumptions of minimal effect in reducing BSIs compared with screening and isolation alone.

The effectiveness estimates for the prevention strategies were derived from a single trial that included mostly community hospitals, although they were largely representative of US hospitals. ${ }^{9}$ Results could vary in hospitals with different patient characteristics, such as proportion of MRSA carriage, or different BSI rates. (The REDUCE MRSA trial focused on community hospital ICUs that may have lower BSI rates than ICUs in academic or specialty cancer care hospitals.) Nevertheless, our sensitivity analyses indicate that the results were robust to changes in those parameters. We did not measure the absolute savings of each strategy compared with no intervention. Among the hospitals in the REDUCE MRSA trial, screening and isolation was the longstanding gold standard, ${ }^{12}$ and that is the strategy to which the other strategies were compared. Any gains attained by screening and isolation before the baseline period were not quantified. Nevertheless, the cost savings of the universal decolonization arm over screening and isolation alone and targeted decolonization is valid. Because we used a static model, we could not explicitly account for possible changes in resistance or herd effects. However, the results of the trial upon which our base case epidemiological assumptions were based incorporated those effects to the extent that they occurred during the period of the trial. The use of a static model also meant that sensitivity analyses (for example, those in which the incidence of BSIs were varied) were not dynamically linked to other parameters, such as MRSA carriage rates, to which they might, in actuality, be related.

Widespread and long-term use of chlorhexidine and mupirocin may engender drug resistance. ${ }^{19,20}$ From the perspec- tive of this study, increased resistance would be tantamount to reduced effectiveness of universal decolonization. However, the effect of universal decolonization on reducing BSIs does not need to be large for that strategy to be preferred. Finally, our perspective was primarily that of the healthcare system and resource use, and so we did not address issues relating to hospital reimbursement.

A strategy of universal decolonization for patients admitted to the ICU would both reduce BSIs and likely reduce healthcare costs compared with strategies of MRSA nares screening and isolation or screening and isolation coupled with targeted decolonization. These findings were robust to a wide range of cost and effectiveness assumptions.

\section{ACKNOWLEDGMENTS}

Financial support. This project was funded by the Healthcare-Associated Infection Program under contract nos. HHSA290201000008I and HHSA29032007T from the Agency for Healthcare Research and Quality (AHRQ), US Department of Health and Human Services, as part of the Developing Evidence to Inform Decisions about Effectiveness (DEcIDE) pro-. gram, and the Centers for Disease Control and Prevention (CDC) Prevention Epicenters Program (1U01 C1000344 to R.P.). The authors of this report are responsible for its content. Statements in the report should not be construed as endorsement by AHRQ or the US Department of Health and Human Services. The findings and conclusions in this report are those of the authors and do not necessarily represent the official position of the CDC. The contents of this publication do not necessarily reflect the views or policies of the Department of Health and Human Services, nor does mention of trade names, commercial products, or organizations imply endorsement by the US government. The authors assume full responsibility of the accuracy and completeness of the ideas presented.

Potential conflicts of interest. S.S.H., E.S., T.R.A., J.H., R.A.W., J.M., M.K.H., and J.B.P. report conducting a trial in which participating hospitals are receiving product contribution from Sage and Molnlycke. This disclosure arose after the conduct and analysis of both the original REDUCE MRSA Trial and this article. E.S. reports receiving consulting fees from $3 \mathrm{M}$ and lecture fees from Sage. M.K.H. conducts other research involving contributed product from Sage. G.T.R. has received research support from Pfizer, GlaxoSmithKline, and Merck Pharmaceuticals. All authors submitted the ICMJE Form for Disclosure of Potential Conflicts of Interest, and the conflicts that the editors consider relevant to this article are disclosed here.

Address correspondence to Susan Huang, MD, MPH, Division of Infectious Diseases, 101 The City Drive South, City Tower, Suite 400, ZC 4081, Orange, CA 92868 (sshuang@uci.edu).

\section{REFERENCES}

1. Vincent JL, Rello J, Marshall J, et al. International study of the prevalence and outcomes of infection in intensive care units. JAMA 2009;302:2323-2329.

2. United States Government Accountability Office. HealthcareAssociate Infections in Hospitals. Report to the Chairman, Committee on Oversight and Government Reform, House of Representatives, 2008.

3. Umscheid CA, Mitchell MD, Doshi JA, Agarwal R, Williams K, Brennan PJ. Estimating the proportion of healthcare-associated infections that are reasonably preventable and the related mortality and costs. Infect Control Hosp Epidemiol 2011;32:101-114. 
4. Lucet JC, Paoletti X, Lolom I, et al. Successful long-term program for controlling methicillin-resistant Staphylococcus aureus in intensive care units. Intensive Care Med 2005;31:1051-1057.

5. Huang SS, Yokoe DS, Hinrichsen VL, et al. Impact of routine intensive care unit surveillance cultures and resultant barrier precautions on hospital-wide methicillin-resistant Staphylococcus aureus bacteremia. Clin Infect Dis 2006;43:971-978.

6. Bleasdale SC, Trick WE, Gonzalez IM, Lyles RD, Hayden MK, Weinstein RA. Effectiveness of chlorhexidine bathing to reduce catheter-associated bloodstream infections in medical intensive care unit patients. Arch Intern Med 2007;167:2073-2079.

7. Ridenour G, Lampen R, Federspiel J, Kritchevsky S, Wong E, Climo M. Selective use of intranasal mupirocin and chlorhexidine bathing and the incidence of methicillin-resistant Staphylococcus aureus colonization and infection among intensive care unit patients. Infect Control Hosp Epidemiol 2007;28:1155-1161.

8. Huskins WC, Huckabee CM, O'Grady NP, et al. Intervention to reduce transmission of resistant bacteria in intensive care. $N$ Engl J Med 2011;364:1407-1418.

9. Huang SS, Septimus E, Kleinman K, et al. Targeted versus universal decolonization to prevent ICU infection. N Engl J Med 2013;368:2255-2265.

10. Climo MW, Yokoe DS, Warren DK, et al. Effect of daily chlorhexidine bathing on hospital-acquired infection. $N$ Engl J Med 2013;368:533-542.

11. Derde LP, Cooper BS, Goossens H, et al. Interventions to reduce colonisation and transmission of antimicrobial-resistant bacteria in intensive care units: an interrupted time series study and cluster randomised trial. Lancet Infect Dis 2013;14:31-39.

12. Perlin JB, Hickok JD, Septimus EJ, Moody JA, Englebright ID, Bracken RM. A bundled approach to reduce methicillin-resistant Staphylococcus aureus infections in a system of community hospitals. J Healthc Qual 2013;35:57-68.

13. Hassan M, Tuckman HP, Patrick RH, Kountz DS, Kohn JL. Cost of hospital-acquired infection. Hosp Top 2010;88:82-89.

14. Roberts RR, Frutos PW, Ciavarella GG, et al. Distribution of variable vs fixed costs of hospital care. JAMA 1999;281:644-649.

15. Nelson RE, Samore MH, Smith KJ, Harbarth S, Rubin MA. Cost-effectiveness of adding decolonization to a surveillance strategy of screening and isolation for methicillin-resistant Staphylococcus aureus carriers. Clin Microbiol Infect 2010;16: 1740-1746.

16. Dasta JF, McLaughlin TP, Mody SH, Piech CT. Daily cost of an intensive care unit day: the contribution of mechanical ventilation. Crit Care Med 2005;33:1266-1271.

17. Graves N. Economics and preventing hospital-acquired infection. Emerg Infect Dis 2004;10:561-566.

18. Graves N, Weinhold D, Tong E, et al. Effect of healthcareacquired infection on length of hospital stay and cost. Infect Control Hosp Epidemiol 2007;28:280-292.

19. Richter SS, Diekema DJ, Heilmann KP, et al. Activities of vancomycin, ceftaroline, and mupirocin against Staphylococcus aureus isolates collected in a 2011 national surveillance study in the United States. Antimicrob Agents Chemother 2014;58(2):740745.

20. Desroches M, Potier J, Laurent F, et al. Prevalence of mupirocin resistance among invasive coagulase-negative staphylococci and methicillin-resistant Staphylococcus aureus (MRSA) in France: emergence of a mupirocin-resistant MRSA clone harbouring mupA. J Antimicrob Chemother 2013;68(8):1714-1717.

21. Kang J, Mandsager P, Biddle AK, Weber DJ. Cost-effectiveness analysis of active surveillance screening for methicillin-resistant Staphylococcus aureus in an academic hospital setting. Infect Control Hosp Epidemiol 2012;33:477-486.

22. Puzniak LA, Gillespie KN, Leet T, Kollef M, Mundy LM. A costbenefit analysis of gown use in controlling vancomycin-resistant Enterococcus transmission: is it worth the price? Infect Control Hosp Epidemiol 2004;25:418-424.

23. Morgan DJ, Pineles $L$, Shardell $M$, et al. The effect of contact precautions on healthcare worker activity in acute care hospitals. Infect Control Hosp Epidemiol 2013;34:69-73.

24. Courville XF, Tomek IM, Kirkland KB, Birhle M, Kantor SR, Finlayson SR. Cost-effectiveness of preoperative nasal mupirocin treatment in preventing surgical site infection in patients undergoing total hip and knee arthroplasty: a cost-effectiveness analysis. Infect Control Hosp Epidemiol 2012;33:152-159.

25. Kilgore M, Brossette S. Cost of bloodstream infections. Am J Infect Control 2008;36:S172-S173. 